INRA Prod. Anim., 2017, 30 (4), 381-394

\section{Enjeux et atouts des productions animales sous signe officiel de qualité pour répondre aux attentes sociétales}

\author{
M. BENOIT', B. MÉDA ${ }^{2}$ \\ ${ }_{1}^{1}$ Université Clermont Auvergne, INRA, Vetagro Sup, UMR Herbivores, 63122, Saint-Genès-Champanelle, France \\ ${ }^{2}$ URA, INRA, 37380, Nouzilly, France \\ Courriel : marc-p.benoit@inra.fr
}

En quoi la production de produits animaux sous signe officiel de qualité permet la fourniture de services spécifiques via la mise en œuvre de principes et de cahiers des charges ? En prenant les exemples de la production d'ovins viande en Agriculture Biologique et de la production de poulets Label Rouge, cet article montre en quoi les cahiers des charges et les pratiques associées influencent les performances techniques, économiques, environnementales et sociétales des systèmes de production et met en évidence les leviers qui permettent d'accroître la production de ces services.

La demande des consommateurs évolue de façon relativement constante vers des produits pour lesquels des garanties sont données par l'existence de cahier des charges encadrant les pratiques. Ces garanties peuvent toucher au bien-être animal, au respect de l'environnement ou aux qualités intrinsèques des produits (Sauveur 1997, Verbeke et Viaene 2000, Siegford et al 2008). Ainsi, des systèmes d'élevage souvent qualifiés d'alternatifs se sont développés. Nous basons notre analyse sur deux d'entre eux : la production de poulets Label Rouge (LR), spécificité française, et la production ovine allaitante en Agriculture Biologique (AB). Ce choix permet $i$ ) d'analyser des modes de production très contrastés (monogastriques et herbivores), ii) de traiter de cas de signes officiels de qualité d'importance très significative en France (poulet LR : 15\% des abattages contrôlés de poulets de chair, SYNALAF 2015) ou en très forte dynamique $(A B$ : doublement de la part de la SAU de 2009 à 2015, Agence Bio 2016a) et iii) d'étudier en parallèle deux filières dédiées à la production de viande en mettant en avant des points d'analyse génériques à ce niveau.

Le tout premier Label Rouge français a été créé en 1965 et concernait un produit avicole : le poulet jaune des Landes. La très forte évolution connue depuis 30 ans a abouti à l'existence en 2016 de plus de 200 cahiers de charges Label Rouge avicoles gérés par l'Institut national de l'ori- gine et de la qualité (INAO), certains étant associés à une Indication Géographique Protégée (figure 1a). Les éléments majeurs des cahiers des charges touchent à l'accès obligatoire à un parcours extérieur pour les animaux (photo1), à l'utilisation de souches à croissance lente associée à un âge d'abattage minimum ( 81 jours pour les poulets de chair) ainsi qu'à la fourniture d'aliments contenant un minimum de céréales (et/ou de leurs coproduits) (tableau 1).

L'AB donne des garanties fortes au consommateur via un cahier des charges uniformisé au niveau européen (Règlements européens 834/2007 - Union Européenne 2007 - et 889/2008 - Union Européenne 2008), avec des conséquences favorables attendues en termes de santé humaine, d'environnement, de bienêtre des animaux et d'un point de vue socio-économique (Reganold et Wachter 2016). Les bases de l'AB sont soutenues par des principes forts (IFOAM 2015) qui rejoignent ceux de l'agroécologie (Dumont et al 2013). Le point central du cahier des charges porte sur l'interdiction d'utiliser des produits chimiques de synthèse (sauf pour la gestion de la santé animale). Un second point est, en élevage, le « lien au sol», qui conduit à utiliser au maximum les ressources de l'exploitation pour alimenter le troupeau et à avoir des surfaces d'épandage suffisantes (tableau 1, photo 2). Ce principe est cohérent avec une approche économique qui pousse les élevages à une forte autonomie alimentaire compte tenu du prix élevé des aliments du commerce en $\mathrm{AB}, 60$ à $70 \%$ plus chers que ceux produits en conventionnel. Le développement de la production en $\mathrm{AB}$ est relativement récent avec une reconnaissance officielle en France en 1980 (figure 1b). La montée en puissance est rapide, mais la part des exploitations en $\mathrm{AB}$ en France reste faible, avec 4,9\% des surfaces fin 2015 (Agence Bio 2016a). La production ovine allaitante $\mathrm{AB}$ représentait un taux comparable, avec $4,8 \%$ des effectifs ovins viande (Agence Bio 2016b).

Des éléments de convergence apparaissent donc à travers les cahiers des charges des poulets LR et de l'élevage en AB : restriction de l'utilisation de certains intrants, obligation d'accès à des surfaces extérieures, absence ou limitation des mutilations. Ces éléments ont des conséquences sur la productivité, l'environnement ou encore le bien-être animal. Notre objectif est, dans un premier temps, d'identifier les services fournis, de mettre en évidence les convergences entre ces deux productions et d'identifier les éventuels services spécifiques à chacune puisqu'elles présentent des caractéristiques propres aux monogastriques d'une part, aux ruminants d'autre part. Notre analyse cherchera à décrire les liens entre cahier des charges, fonctionnement des systèmes d'élevage (et pratiques), et services fournis. Cette approche nous permettra de mettre en évidence les leviers mobilisables pour améliorer ces services. 
Figure 1. Dates et chiffres clés pour a) les productions avicoles Label Rouge (LR) et b) l'Agriculture Biologique (AB) en France.

(a)

\section{5}

Création du premier

Label Rouge en France

«Poulet jaune des Landes »
1998

Apparition

du Label Rouge pour les oeufs

de consommation

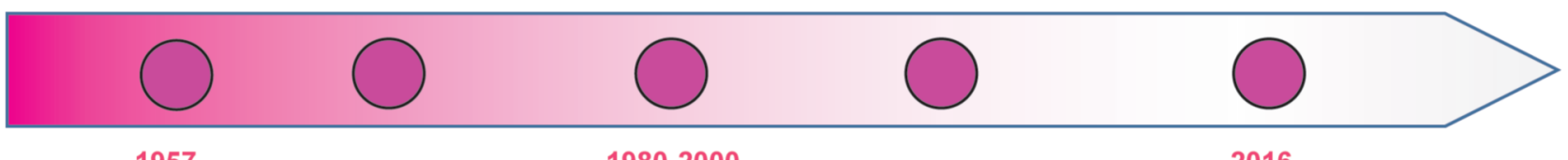

1957

Appellation d'Origine Contrôlée

"Volailles de Bresse "
Augmentation constante de la production de volailles de chair sous Label Rouge (+360\% sur la période)
Plus de 200 cahiers des charges

Label Rouge et 40 IGP pour les volailles de chair. Les poulets Label Rouge représentent $15 \%$ des abattages contôlés en France

(b)

\section{2}

Création de l'IFOAM :

(Fédération internationale des mouvements de l'AB)
1980

Loi d'Orientation Agricole (France) : il est question de l'AB et réflexion sur un cahier des charges $A B$
2007

Cahier des charges

européen de l'AB

$N^{\circ} 834 / 2007$

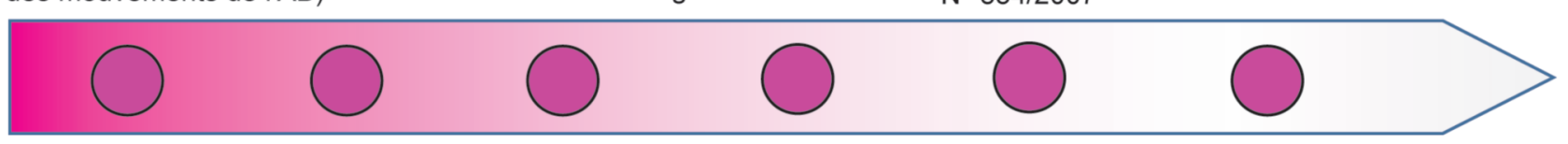

1978

Création de la FNAB

(Fédération Française de l'AB)

\section{0}

Surface en $A B$ en France :

$0,8 \%$ du total fin 2015

Surface AB France : $4,9 \%$ du total

Cheptel ovin viande $A B: 4,8 \%$ du total

Photo 1. Le cahier des charges Label Rouge impose la fourniture d'un accès extérieur aux poulets au plus tard à 6 semaines d'âge. L'aménagement du parcours (haies, arbres...) est essentiel pour permettre aux animaux d'explorer toute la surface disponible. Le parcours fournit en outre aux animaux de l'ombre, une protection contre les prédateurs, voire un complément alimentaire (protéines, plantes " médicinales ») et peut permettre de dégager des revenus supplémentaires (bois, vergers).
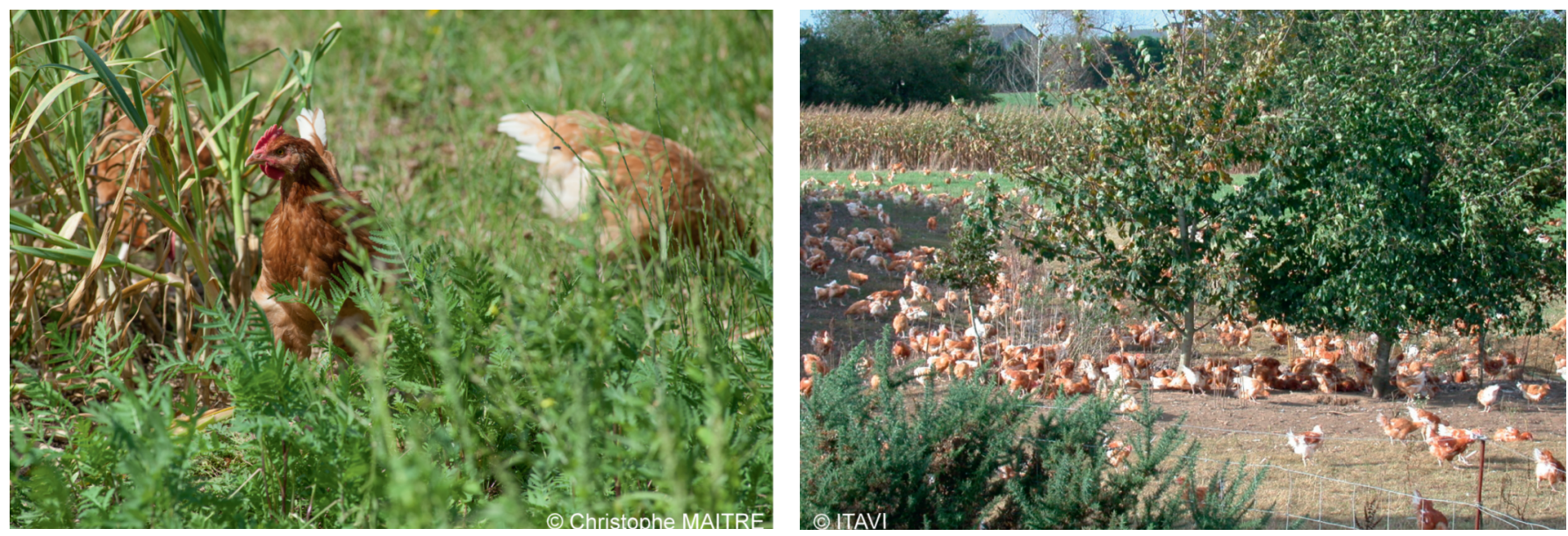

Photo 2. La recherche d'une forte autonomie alimentaire en Agriculture Biologique conduit à maximiser le pâturage des animaux adultes ou des jeunes et à renforcer la culture de céréales sur l'exploitation, y compris en zone de montagne (triticale; Lozère, 1100 m d'altitude), ce qui peut aussi permettre de tendre vers une autonomie totale en paille (litière).
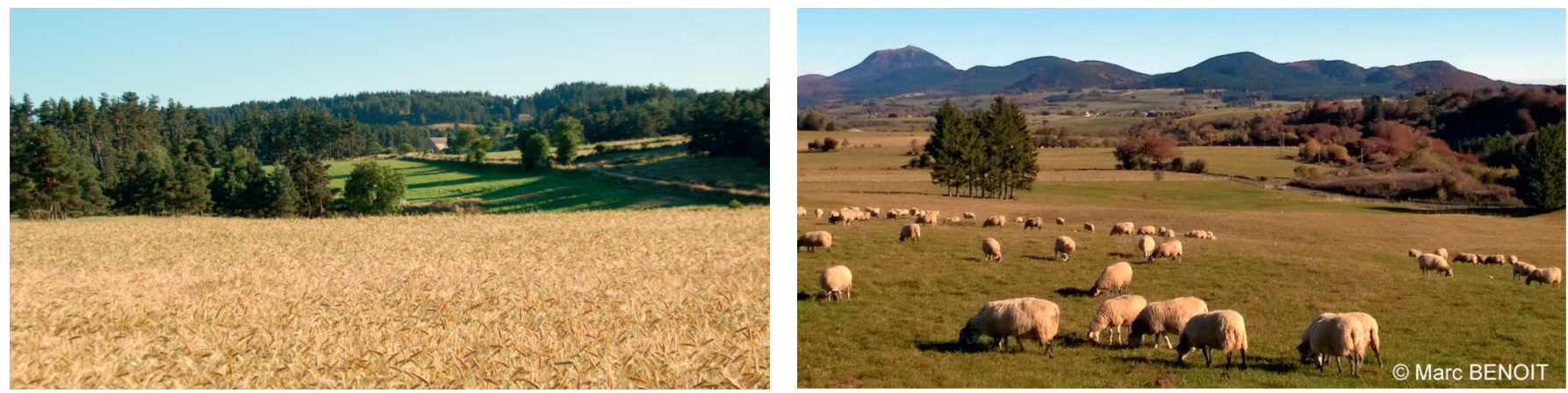
Tableau 1. Principales caractéristiques des productions de poulets Label Rouge (LR) et d'ovins biologiques (AB).

Rouge : cadre réglementaire (1), (2) ; noir : contraintes fixées par des organisations de production ou par les éleveurs eux-mêmes ; $(\mathrm{P})$ : principes et préconisations.

\begin{tabular}{|c|c|c|}
\hline & Poulets LR & Ovins AB \\
\hline $\begin{array}{l}\text { Génétique } \\
\text { et animaux }\end{array}$ & - Souches à croissance lente & $\begin{array}{l}\text { - Races locales ou rustiques }(P) \\
\text { - Achat des animaux produits en AB sinon } \\
\text { maximum de } 20 \% \text { du cheptel adulte (femelles) }\end{array}$ \\
\hline Alimentation & $\begin{array}{l}\text { - Aliments composés incorporant un minimum de } \\
\text { céréales (et coproduits) : } 50 \% \text { avant } 28 \text { j d'âge } \\
\text { puis } 75 \% \text { à partir de } 29 \text { j d'âge } \\
\text { - Alimentation d'origine locale encouragée }\end{array}$ & $\begin{array}{l}\text { - Production au minimum de } 60 \% \text { des aliments } \\
\text { sur l'exploitation (ou issus de fermes en } A B \text { de la } \\
\text { région) } \\
\text { - Allaitement maternel pendant un minimum de } 45 \\
\text { jours } \\
\text { - Au moins } 60 \% \text { de la matière sèche composant } \\
\text { la ration journalière provient de fourrages } \\
\text { grossiers, frais, séchés ou ensilés } \\
\text { - OGM, acides aminés de synthèse, facteurs de } \\
\text { croissance interdits }\end{array}$ \\
\hline Reproduction & & $\begin{array}{l}\text { - Insémination artificielle et traitements lumineux } \\
\text { autorisés. } \\
\text { - Synchronisation des chaleurs et transfert } \\
\text { d'embryon interdits. }\end{array}$ \\
\hline Age d'abattage & - 81 jours minimum & \\
\hline $\begin{array}{l}\text { Surfaces, } \\
\text { Parcours/Pâturage }\end{array}$ & $\begin{array}{l}\text { - Accès à un parcours au plus tard à } 6 \text { semaines } \\
\text { d'âge } \\
\text { - } 2 \text { m² minimum par animal (surface illimitée pour }^{2} \text { l'appellation « en liberté ») } \\
\text { - Vide sanitaire de } 7 \text { semaines minimum entre } \\
\text { deux lots } \\
\text { - Présence d'arbres sur le parcours ( } 20 \text { arbres } \\
\text { minimum) } \\
\text { - Aménagement des parcours (nombres } \\
\text { d'espèces végétales, zones d'implantation, } \\
\text { linéaires minimum) }\end{array}$ & $\begin{array}{l}\text { - Chargement limité pour ne pas excéder } 170 \text { kg } \\
\text { N/ha/an (soit } 2 \text { UGB/ha) } \\
\text { - Épandage des effluents sur des terres en AB } \\
\text { - Accès des animaux aux pâturages quand les } \\
\text { conditions pédoclimatiques le permettent }\end{array}$ \\
\hline Bâtiments & $\begin{array}{l}\text { - Surface maximum : } 400 \mathrm{~m}^{2} / \text { bâtiment } \\
\text { (4 bâtiments maximum sur l'exploitation) } \\
\text { - Densité animale maximum : } 11 \text { animaux } / \mathrm{m}^{2}\end{array}$ & $\begin{array}{l}\text { - } 1,5 \mathrm{~m}^{2} \text { minimum par brebis } \\
-0,35 \mathrm{~m}^{2} \text { minimum par agneau (+ aire extérieure } \\
\text { d'exercice de } 0,5 \mathrm{~m}^{2} \text { minimum) }\end{array}$ \\
\hline Santé animale & - Utilisation raisonnée (voire nulle) d'antibiotiques & $\begin{array}{l}\text { - Prévention prioritaire, recours en premier lieu à } \\
\text { homéopathie, phytothérapie, oligo-éléments }(P) \\
\text { - Pas de limite pour vaccinations et traitements } \\
\text { antiparasitaires } \\
\text { - Autres traitements limités à 3/brebis/an et } 1 \text { pour } \\
\text { agneaux (sinon perte de label AB ou conversion } 6 \\
\text { mois). } \\
\text { - Traitements allopathiques : délai d'attente } \\
\text { doublé par rapport au délai légal et, si absence, } \\
\text { minimum de } 48 \text { h }\end{array}$ \\
\hline Bien-être animal & & $\begin{array}{l}\text { - Absence ou limitation des mutilations } \\
\text { (castration, caudectomie...) }\end{array}$ \\
\hline $\begin{array}{l}\text { Productions } \\
\text { végétales }\end{array}$ & & $\begin{array}{l}\text { - Molécules de synthèse interdites (engrais et } \\
\text { pesticides) }\end{array}$ \\
\hline Commercialisation & & $\begin{array}{l}\text { - Relations de proximité avec le consommateur } \\
\text { encouragées }(P)\end{array}$ \\
\hline
\end{tabular}

(1) Poulets LR : Notice technique INAO « volailles fermières de chair label rouge ».

${ }^{(2)}$ Réglementation en $A B$ concernant les petits ruminants : règlements européens $n^{\circ} 834 / 2007$ et $n^{\circ} 889 / 2008$. 
Figure 2. Bouquet de services fournis par la production ovine biologique (représentation conceptuelle "Grange » décrite par Duru et al 2017). Les éléments d'analyse sont précisés dans le tableau 2.

Le pentagone central figure un système ou un territoire d'élevage. Celui-ci intègre certains éléments paysagers (haies) et les activités agro-industrielles sur lesquelles s'appuie l'élevage. Ce territoire d'élevage est décrit par ses caractéristiques : espèces animales, source de l'alimentation (animal sur herbe : pâturage et fourrages, sinon à base d'aliments concentrés dont l'ensilage de maïs). Le mode d'usage des sols est suggéré par un parcellaire à deux nuances de vert pour symboliser la diversité des prairies permanentes et la présence de temporaires, et à deux nuances de jaune pour représenter la diversité des rotations culturales.

Au sein du territoire, les systèmes d'élevage interagissent avec cinq interfaces : les marchés, le travail et l'emploi, les intrants, l'environnement et le climat, et les enjeux sociaux et culturels. Des pictogrammes explicités ci-dessous symbolisent les éléments concernés par ces interfaces. La nature et l'ampleur des effets sont représentées par une flèche sortante plus ou moins large et dont la couleur indique que ceux-ci sont positifs (flèche verte), négatifs (rouge) ou mitigés (hachures) ; dans ce cas, l'effet dominant borde la flèche. Sur l'interface " travail et emploi », nous distinguons les emplois directs en élevage des emplois indirects (IAA, tourisme, flèche vers personnage en blanc). Les flèches entrantes indiquent l'existence de pressions liées à l'utilisation d'intrants exogènes (qui symbolisent les effets délocalisés de l'élevage), et sur l'interface " marchés » des opportunités liées aux circuits courts. Sur l'interface " environnement et climat ", les flèches entrantes indiquent que les systèmes d'élevage bénéficient de services intrants (flèche verte) ou subissent la pression de facteurs environnementaux (flèches rouges : prédation, avifaune sauvage vecteur de la grippe aviaire...).

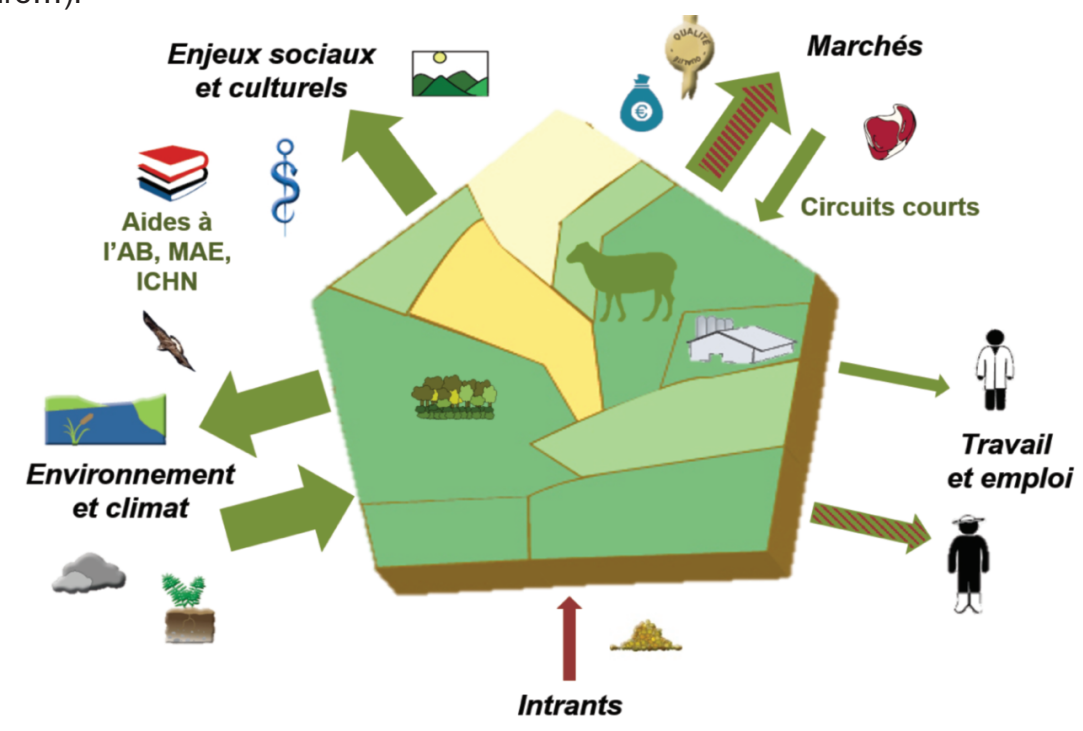

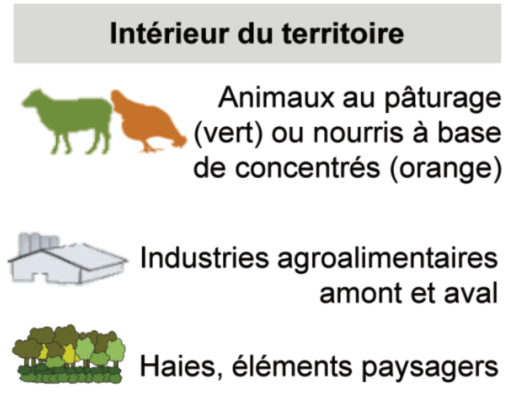

Environnement et climat

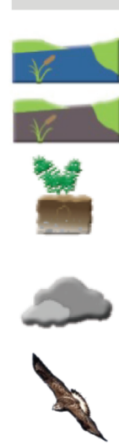

Eau de bonne qualité

Eutrophisation

Qualité des sols

Emission de GES et de particules fines dans l'air

Biodiversité

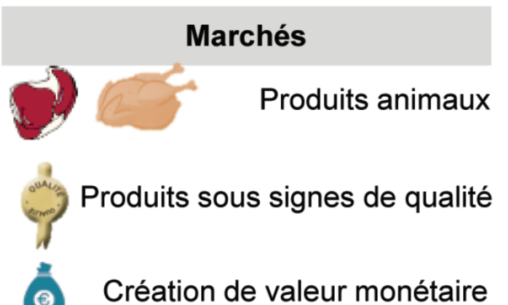

Intrants

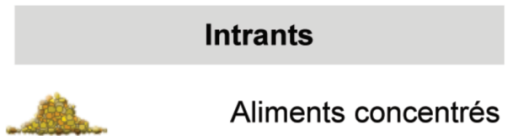

Engrais, pesticides, énergie

\section{Enjeux sociaux et culturels}

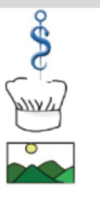

Aspects sanitaires

Patrimoine gastronomique

Esthétique paysagère

Aspects réglementaires et juridiques

Travail et emploi




\section{1 / Incidences des cahiers des charges et des pratiques associées sur la production de services}

Les principaux éléments du cadre réglementaire des productions de poulets LR et d'ovins AB sont récapitulés dans le tableau 1. En plus de points de réglementation générale, d'autres peuvent être liés à des cahiers des charges spécifiques (i.e. propres à certaines organisations de production, pour les poulets LR), à l'appréciation de l'éleveur, ou à des principes généraux.

Selon la représentation conceptuelle développée par Duru et al (2017), ce numéro, nous synthétisons les services rendus par ces deux productions (ovins $\mathrm{AB}$ et poulets LR) dans les figures 2 et 3 , les tableaux respectifs d'accompagnement des schémas (tableaux 2 et 3 ) reprenant quelques services majeurs.

\section{1 / Services d'approvisionnement}

En production ovine allaitante, la productivité numérique (nombre d'agneaux produits par brebis et par an) est un élément majeur de la performance économique (Benoit et al 1999, Bellet et Morin 2005). En production AB, cette productivité peut être plus faible que celle des systèmes conventionnels de 14 à $18 \%$ (Bellet et al 2016) en raison notamment i) de l'interdiction des traitements hormonaux pour le désaisonnement des femelles reproductrices (tableau 1), ii) de la recherche d'optimum entre productivité et coûts de production (non mise en œuvre d'un système « accéléré » avec 3 agnelages en 2 ans, Benoit et al 2009), et iii) de la priorité mise par les éleveurs sur d'autres objectifs (vente en circuits courts, autres activités...).

L'interdiction des traitements hormonaux a en outre une incidence sur la régularité d'apport des agneaux sur le marché ; c'est en particulier vrai dans les zones de plaine où les races herbagères très saisonnées sont majoritaires (mises bas entre janvier et juin). Cette interdiction empêche en outre le recours à l'insémination artificielle. Par ailleurs, le coût très élevé des concentrés biologiques est une forte incitation à engraisser les agneaux totalement ou partiellement au pâturage, alors que cette pratique est minoritaire dans les systèmes conventionnels. Bien que les niveaux de croissance des agneaux engraissés à l'herbe (âge à la vente de 150 à 180 jours) soient inférieurs à ceux des agneaux engraissés en bergerie (âge à la vente de 120 à 130 jours), l'engraissement à l'herbe a toutefois une incidence positive sur la qualité nutritionnelle de la viande (acides gras favorables à la santé humaine) (Aurousseau et al 2004, Srednicka-Tober et al 2016) malgré un effet potentiellement négatif d'un point de vue organoleptique avec des teneurs en scatole augmentées lorsque la consommation de légumineuses est importante (Prache et al 2009). Enfin, les mises bas de fin d'automne et début d'hiver donnent lieu à un engraissement systématique en bergerie avec des concentrés ce qui engendre un coût de production important. Elles gardent en général un intérêt si elles procurent un argument commercial (prix élevé des agneaux à cette saison ; contrat annuel pour une bonne valorisation moyenne des agneaux).
Afin d'atteindre le niveau de qualité des produits requis pour l'obtention du Label Rouge (LR), la production de poulets LR repose sur l'élevage de poulets à croissance lente et sur un âge d'abattage minimal environ deux fois plus élevé qu'en production conventionnelle (81 contre 30 à 50 jours, tableau 1 ). Ainsi, les différences de génotype conduisent à une efficacité alimentaire réduite avec des indices de consommation entre 3 et 4 contre 1,7 et 2 pour des poulets conventionnels (Sauveur 1997, Fanatico et al 2007) ce qui se répercute sur le coût de production, et donc sur le prix payé par le consommateur. Cependant, l'âge d'abattage élevé a une incidence sur la qualité de ces produits, essentiellement basée sur une différenciation vis-à-vis des volailles conventionnelles en termes de teneur en lipides totaux (plus faible), de fermeté et de couleur de la chair (Sauveur 1997, Chartrin et al 2005, Fanatico et al 2007). Ces caractéristiques, permettent à ces produits de justifier, après dégustation par des jurys et panels de consommateurs, de qualités gustatives supérieures. Les consommateurs sont très sensibles à cette différenciation, et citent le goût comme principal critère de qualité de ces produits. Ainsi, selon Tavoularis et al (2007), 60\% d'entre eux se disent prêt à payer plus cher pour acheter ces volailles " meilleures au goût». Ainsi, le surcout actuel de ces produits (environ $+40 \%$ sur la période 2003-2015 pour un poulet entier vendu en GMS par rapport à un produit conventionnel selon l'Observatoire de la formation des prix et des marges des produits alimentaires) semble être bien accepté par le consommateur, au vu de la bonne santé de la filière.

Figure 3. Bouquet de services fournis par la production de poulets Label Rouge (représentation conceptuelle «Grange » décrite par Duru et al 2017). Définition des pictogrammes à la figure 2 et analyse dans le tableau 3.

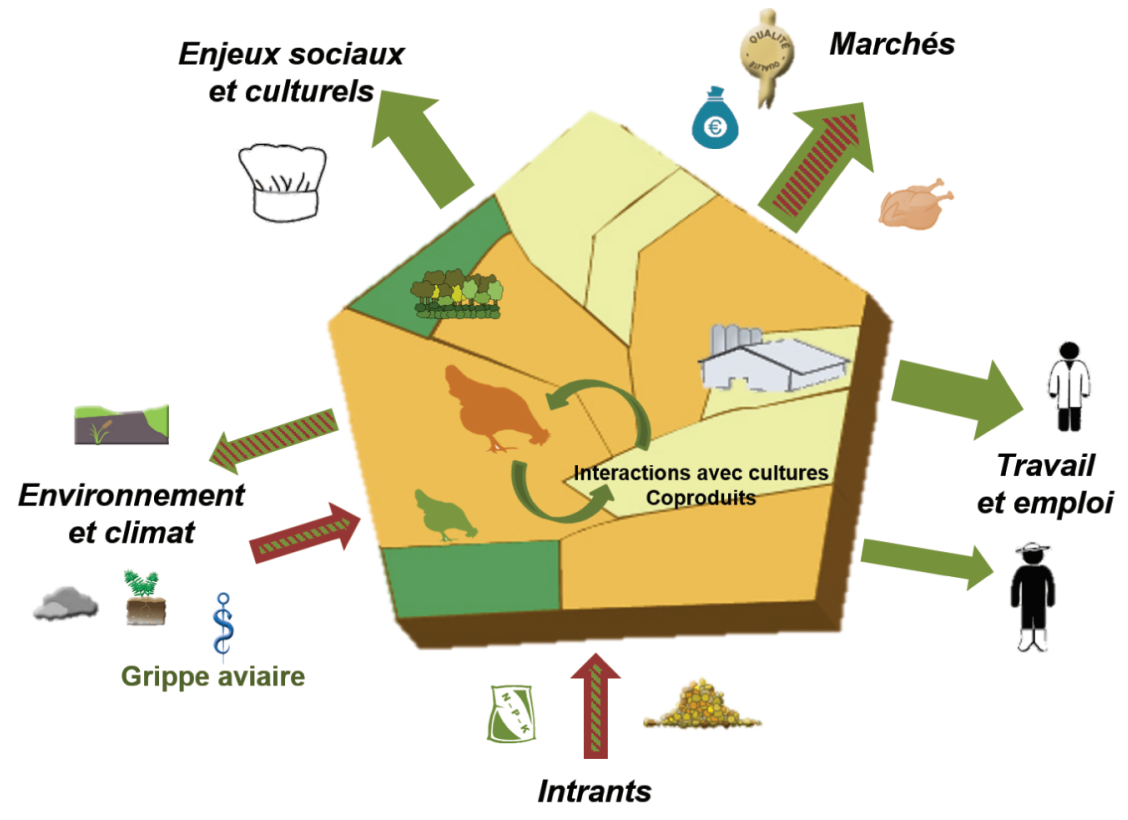


Tableau 2. Éléments d'analyse des principaux services fournis par la production ovine allaitante biologique et présentés en figure 2.

\begin{tabular}{|c|c|c|}
\hline $\begin{array}{l}\text { Interfaces } \\
\text { étudiées }\end{array}$ & Effets positifs & Effets négatifs \\
\hline Intrants & - Niveau d'autonomie alimentaire élevée & $\begin{array}{l}\text { - Prix élevé des aliments Bio } \\
\text { - Limitation des intrants par prix et cahier } \\
\text { des charges (aliments, engrais, produits } \\
\text { vétérinaires) } \\
\text { - Énergie pour mécanisation et stocks }\end{array}$ \\
\hline Travail et emploi & $\begin{array}{l}\text { - Emplois directs en élevage (+ d'emploi par } \\
\text { exploitation qu'en conventionnel) } \\
\text { - Satisfaction au travail } \\
\text { - Emplois indirects (circuits courts) }\end{array}$ & $\begin{array}{l}\text { - Organisation de la commercialisation } \\
\text { (faibles volumes) } \\
\text { - Charge de travail élevée }\end{array}$ \\
\hline Marchés & $\begin{array}{l}\text { - Produits avec potentiel de valeur ajoutée } \\
\text { (Label bio, circuits courts) }\end{array}$ & $\begin{array}{l}\text { - Prix élevé pour le consommateur } \\
\text { - Risque de valorisation insuffisante qui } \\
\text { ne couvre pas les coûts de production } \\
\text { - Saisonnalité de la production }\end{array}$ \\
\hline $\begin{array}{l}\text { Enjeux sociaux } \\
\text { et culturels }\end{array}$ & $\begin{array}{l}\text { - Image de la production } \\
\text { - Consentement à payer pour des impacts } \\
\text { sociétaux positifs (santé, bien-être animal, } \\
\text { environnement) } \\
\text { - Gestion intégrée de la santé animale } \\
\text { - Valeur nutritionnelle des produits à l'herbe }\end{array}$ & $\begin{array}{l}\text { - Vigilance par rapport à la qualité } \\
\text { sensorielle de la viande d'agneaux } \\
\text { (scatole) }\end{array}$ \\
\hline $\begin{array}{l}\text { Environnement } \\
\text { et climat }\end{array}$ & $\begin{array}{l}\text { - Préservation de la qualité des eaux (absence } \\
\text { de pesticides de synthèse et d'N minéral). } \\
\text { - Peu de GES indirects (moins d'intrants, dont } \\
\text { engrais azoté) } \\
\text { - Biodiversité " commune » (prairies, bocage) et } \\
\text { domestique (races adaptées) } \\
\text { - Services intrants : légumineuses, teneur en } \\
\text { matière organique des sols, agroforesterie }\end{array}$ & $\begin{array}{l}\text { - Émissions de } \mathrm{CH}_{4} / \mathrm{kg} \text { produit supérieure } \\
\text { par rapport aux produits conventionnels } \\
\text { (productivité animale plus faible) }\end{array}$ \\
\hline
\end{tabular}

Tableau 3. Éléments d'analyse des principaux services fournis par la production Poulets Label Rouge et présentés en figure 3.

\begin{tabular}{|c|c|c|}
\hline $\begin{array}{l}\text { Interfaces } \\
\text { étudiées }\end{array}$ & Effets positifs & Effets négatifs \\
\hline Intrants & $\begin{array}{l}\text { - Moindre utilisation de soja (besoins } \\
\text { protéiques plus faibles) qui permet une plus } \\
\text { grande valorisation de coproduits et de } \\
\text { céréales produites localement }\end{array}$ & $\begin{array}{l}\text { - Besoins en aliments accrus par une durée } \\
\text { d'élevage plus longue } \\
\text { - Effets délocalisés pour la production d'aliments } \\
\text { concentrés } \\
\text { - Équipements }\end{array}$ \\
\hline Travail Emploi & $\begin{array}{l}\text { - Nombreux emplois locaux majoritairement } \\
\text { dans filière bien organisée : accouvage, } \\
\text { fabrication d'aliments, abattage, distribution } \\
\text { - Satisfaction au travail pour l'éleveur }\end{array}$ & \\
\hline Marchés & $\begin{array}{l}\text { - Produits sous signes de qualité ( } 200 \text { Labels } \\
\text { Rouge avicoles) } \\
\text { - Bois/fruits si agroforesterie }\end{array}$ & $\begin{array}{l}\text { - Valeur ajoutée inégalement répartie dans la } \\
\text { filière }\end{array}$ \\
\hline $\begin{array}{l}\text { Enjeux sociaux } \\
\text { et culturels }\end{array}$ & $\begin{array}{l}\text { - Image positive de la production } \\
\text { - Traçabilité par label } \\
\text { - Consentement à payer pour qualité, image et } \\
\text { bien-être animal } \\
\text { - Qualité gustative (fermeté chair) et } \\
\text { nutritionnelle (moindre teneur en lipides) }\end{array}$ & \\
\hline $\begin{array}{l}\text { Environnement } \\
\text { et climat }\end{array}$ & $\begin{array}{l}\text { - Adéquation entre fumier produit et capacités } \\
\text { d'épandage, optimisation recyclage déjections } \\
\text { - Diversité domestique (souches à croissance } \\
\text { lente) et biodiversité sur les parcours } \\
\text { - Services intrants des parcours : ombrage, } \\
\text { picorage. Séquestration de carbone par les } \\
\text { parcours (et arbres) }\end{array}$ & $\begin{array}{l}\text { - Risques de pollution (sols, eau) si mauvaise } \\
\text { utilisation du parcours par les animaux } \\
\text { - Émissions GES plus élevées du fait de la } \\
\text { moindre efficacité alimentaire } \\
\text { - Risques sanitaires (avifaune sauvage vecteur } \\
\text { de grippe aviaire) et de prédation. }\end{array}$ \\
\hline
\end{tabular}


En outre, dans les systèmes d'agroforesterie, le revenu issu de la production de viande de poulet peut être complété par la production de bois d'œuvre ou de chauffage, ou de fruits (Clark et Gage 1996, Lubac et al 2003, Lavigne et al 2012). Ainsi, Lubac et al (2014) rapportent dans une enquête que les essences implantées sur près de la moitié des parcours enquêtés $(47 \%)$ sont exploités pour une valorisation, principalement en bois de chauffage et bois d'œuvre. L'association animaux/ couvert arboré est généralement positive pour les deux productions (ombre et protections contre les prédateurs $v s$ fertilisation organique par les déjections). Par exemple, Dubois et al (2008) ont montré que l'association entre oies et noyers améliore la croissance des arbres de 6\% et la production de noix de $26 \%$. Toutefois, il est difficile d'évaluer la contribution de tels systèmes à la production nationale, bien qu'aujourd'hui soutenus par les pouvoirs publics (mesures agro-environnementales).

\section{2 / Services et impacts environ- nementaux}

En production ovine allaitante, la productivité numérique a un impact direct (via un effet « dilution ») sur les émissions de Gaz à Effet de Serre (GES) par $\mathrm{kg}$ de carcasse, en liaison avec les émissions de méthane entérique (Benoit et al 2010) qui proviennent essentiellement des brebis. Les plus faibles niveaux de productivité numérique observées en $\mathrm{AB}$ peuvent donc avoir un impact négatif à ce niveau. La moindre vitesse de croissance des agneaux $\mathrm{AB}$, en lien avec la stratégie de réduction de l'utilisation de concentrés, l'engraissement à l'herbe et une vente tardive (150-200 jours), peut être un léger handicap, le " poids » du troupeau de mère étant cependant prépondérant à ce niveau. Au final, compte tenu de la forte réduction des intrants en $\mathrm{AB}$, les émissions brutes de GES (équivalents- $\mathrm{CO}_{2}$, approche par Analyse de Cycle de Vie - ACV) par kg de carcasse produit sont cependant proches de celles des élevages conventionnels (-3\% en moyenne selon Benoit et Dakpo 2012). Le remplacement des engrais minéraux de synthèse par l'implantation de légumineuses dans les prairies joue un rôle fondamental, en limitant la consommation d'énergie indirecte de même que les émissions de $\mathrm{N}_{2} \mathrm{O}$ à l'épandage. Si l'on prend en compte la séquestration du carbone dans les prairies, les émissions nettes de GES par kg de carcasse sont inférieures de $7 \%$ en AB (Benoit et Dakpo 2012). Sur cette base, si l'on considère le prix de la viande supérieur en $A B$, les émissions nettes de GES par $€$ de produit sont encore plus favorables à l'AB (-16\% vs conventionnel, sur la base d'une plusvalue au $\mathrm{kg}$ de $10 \%$ ). Cet indicateur revient à rapporter les émissions de
GES à la viande mais aussi aux externalités positives produites pouvant justifier le prix plus élevé (biodiversité, qualité de la ressource en eau...). Enfin, il faut rappeler la non utilisation de pesticides de synthèse en $\mathrm{AB}$ (sur la ferme ou sur les lieux de production des ressources achetées, tableau 1) qui conduit à une préservation des ressources en eau et de la biodiversité, et prévient de dommages potentiels sur la santé humaine (Reganold et Wachter 2016).

Pour les poulets LR, l'utilisation de souches à croissance lente ainsi qu'un âge à l'abattage élevé (tableau 1) se traduisent par une efficacité alimentaire réduite ce qui augmente les impacts environnementaux (évalués par ACV et exprimés par kg de poulet) qui seraient ainsi supérieurs de 20 à $60 \%$ (contribution au changement climatique, eutrophisation, acidification...). En revanche, les conclusions sont inversées avec des impacts exprimés par euro de valeur produite de 15 à $40 \%$ plus faibles pour les poulets LR, qui sont vendus plus cher, en lien avec leurs qualités gustatives supérieures et leurs coûts de production plus élevés (da Silva et al 2014).

La recherche du « lien au sol» conduit les éleveurs ovins à maximiser l'utilisation des ressources de la ferme en cultivant, si possible, des céréales pour le troupeau, ce qui peut conduire à un déstockage de carbone via le labour. Il faut cependant noter qu'en conventionnel le bilan carbone est comparable si l'on considère le travail du sol de surfaces hors exploitation (voire hors France), le concentré étant le plus fréquemment acheté, en particulier par les éleveurs des zones défavorisées où se situent fréquemment les élevages. Cette démarche de recherche d'autonomie alimentaire en $\mathrm{AB}$ tend à « boucler» les cycles minéraux (bilan azoté apparent le plus souvent proche de 0 ) et conduit les éleveurs à mettre en place des rotations de cultures qui apportent un cortège de biodiversité associée (flore messicole, parasites et prédateurs associés, avifaune). La recherche d'autonomie alimentaire peut également conduire à la pratique du pâturage des brebis sur des surfaces pastorales très extensives voire à du pâturage hivernal. Ce type de conduite, non spécifique de l'AB mais très adapté, contribue au maintien de milieux ouverts, et permet de diminuer les émissions de GES liées d'une part à l'accumulation du fumier en bâtiment $\left(\mathrm{CH}_{4}\right)$ et à son épandage $\left(\mathrm{N}_{2} \mathrm{O}\right)$, d'autre part à la mécanisation associée à la récolte, au transport de fourrages et de paille, à l'épandage du fumier (matériel et fuel ; $\mathrm{CO}_{2}$ par approche ACV) (Pottier et al 2009).

En production de poulets LR, le parcours est un élément essentiel du système d'élevage, notamment pour permettre aux animaux d'exprimer leurs comportements naturels d'exploration. Sa surface est réglementée selon les cahiers des charges (tableau 1) et les caractéristiques du parcours conditionnent fortement le comportement des volailles. Ainsi, des sorties plus fréquentes et davantage d'animaux sont observés sur le parcours lorsque celui-ci est aménagé avec des arbres, arbustes ou bosquets, et lorsque ces éléments sont distribués sur l'ensemble du parcours (Lubac et al 2003, Zeltner et Hirt 2003 et 2008). Des quantités importantes de nutriments (azote, phosphore, cuivre, zinc) peuvent en revanche s'accumuler sur de petites surfaces, notamment à proximité des bâtiments, lorsque les poulets n'exploitent pas l'ensemble du parcours ; ceci accroît les risques de lessivage et d'émissions gazeuses. À l'inverse, sur des parcours aménagés, la répartition homogène des déjections favorise le recyclage des éléments excrétés par le sol et la végétation (Kratz et al 2004, Dekker et al 2012, Méda et al 2012). Enfin, même si le stockage de carbone est encore très peu étudié pour les volailles en plein-air, selon Méda et al (2012), le potentiel de séquestration par les parcours semble réel (environ $700 \mathrm{~kg} \mathrm{C} / \mathrm{ha} / \mathrm{an}$ ). Par ailleurs, l'aménagement des parcours (arbres, haies, bosquets) contribue à l'intégration paysagère des élevages et donc leur acceptabilité sociale, notamment quand ils sont à proximité de zones d'habitation (Chevalier et al 2015). Les cahiers des charges LR établissent en outre des normes concernant l'aménagement des parcours avec l'obligation d'implanter des arbres d'essence locale en nombre suffisant, voire un linéaire de haies minimum (tableau 1). Ainsi, cette diversité d'essences végétales (pouvant aller jusqu'à une vingtaine sur un même parcours d'après Lubac et al (2014)) pourrait également bénéficier aux populations d'invertébrés qui contribuent à la pollinisation ou la décomposition et le recyclage de la matière organique et servent parfois de complément protéique aux volailles (van de Weerd et al 2009). Enfin, dans le cas de systèmes agroforestiers, les poulets peuvent exercer un contrôle biologique sur les populations de ravageurs et d'adventices en les consommant (Clark et Gage 1996, Lavigne et al 2012), ce qui permet de réduire l'usage de pesticides, et donc les risques pour l'environnement (dégradatation de la qualité de l'eau, effets néfastes sur la biodiversité).

\section{3 / Impacts socio-économiques et activités territoriales}

La dimension des troupeaux est généralement inférieure en AB par rapport aux élevages conventionnels, du fait de structures de production de dimension inférieure et d'une diversification des acti- 
vités plus forte (Laignel et Benoit 2004). Par ailleurs, globalement, les exploitations en $\mathrm{AB}$ emploient en moyenne plus de main-d'œuvre que les exploitations de même type en conventionnel : Massis et Hild (2016) montrent que les exploitations en $\mathrm{AB}$ comptent en France 0,08 UTA supplémentaire, après correction de divers facteurs, dont la présence ou non de vente directe. Pour un autre type de production de ruminants, les bovins laitiers, Garambois et Devienne (2010) montrent, dans une approche territoriale, que les exploitations en $\mathrm{AB}$ ou à faible niveau d'intrants créent plus d'emplois, malgré des volumes mis en marché réduits, avec la spécificité de dégager fréquemment une valeur ajoutée supplémentaire via la commercialisation en circuit court. Le lien avec le consommateur est inscrit dans les principes de l'AB (IFOAM 2015) ; cette proximité permet un dialogue donnant l'opportunité de rappeler les contraintes de saisonnalité de produits comme l'agneau engraissé à l'herbe. La diversification des systèmes de production (conséquence des principes de base de l'AB et de l'agroécologie) est cohérente avec ce mode de commercialisation car il permet le développement d'une économie de gamme (large éventail de produits proposés à la vente), avec des impacts très favorables en termes de satisfaction du consommateur, de maintien de valeur ajoutée sur le territoire, de production locale (impact du transport réduit), voire de préservation de la biodiversité et des paysages.

Les ateliers de poulets LR sont de taille modeste puisque la réglementation impose un nombre maximal de poulets présents sur l'exploitation (17 600), élevés dans des bâtiments de $400 \mathrm{~m}^{2}$ maximum (4 bâtiments au maximum ; tableau 1). De plus, il s'agit souvent d'ateliers complémentaires au sein des exploitations. Cependant, les filières poulets LR sont fortement ancrées dans leurs territoires et génèrent de nombreux emplois locaux puisque concentrant la majorité des maillons de la filière (accouvage, fabrication d'aliment, élevage, abattage et distribution locale), et ce, malgré des tailles différentes de filières entre labels et territoires. Ainsi, en région Pays-dela-Loire, première région productrice de volailles LR, Protino et al (2015) estiment que cette filière génère près de 10000 emplois locaux (dont 2400 éleveurs environ), mais soulignent que la valeur ajoutée, bien que majoritairement produite sur le territoire, n'est pas correctement répartie entre les maillons de la filière, car majoritairement captée par la grande distribution. Au vu du poids que celle-ci représente dans la commercialisation des poulets LR (environ $90 \%$ des poulets LR entiers sont vendus en GMS et hard discount), il apparaît aujourd'hui difficile d'envisager des solutions pour mieux répartir la valeur ajoutée au sein de la filière. Enfin, hormis pour des produits issus de grandes organisations de production, disponibles sur tout le territoire via la grande distribution, la majorité des poulets LR produits en France sont commercialisés dans les territoires où ils sont produits (ITAVI, communication personnelle).

\section{4 / Santé et bien-être des animaux, santé publique}

En production de poulet LR, la tendance est à une utilisation raisonnée des antibiotiques (tableau 1), et certains produits proposés par la grande distribution (voire vendus sous marque distributeur) s'affichent sans antibiotiques (tableau 1). En production ovine $\mathrm{AB}$, l'interdiction d'utilisation des produits chimiques de synthèse évite la diffusion de molécules de synthèse dans les milieux et les effets associés sur la santé publique (Bourguet et Guillemaud 2016). La santé des animaux dans cette production représente un fort enjeu. En effet, les ovins sont une espèce particulièrement sensible au parasitisme, en particulier les agneaux (Cabaret et Nicourt 2009). En élevage conventionnel, l'engraissement très majoritaire en bergerie limite ce problème, que l'on retrouve de façon importante en $\mathrm{AB}$ où les agneaux ont plus fréquemment accès au pâturage. A contrario, les principes de l'AB visent à anticiper les problèmes de santé, et en particulier les infestations parasitaires des animaux. Les éleveurs utilisent globalement moins de traitements anthelminthiques chimiques, ces molécules étant ainsi moins diffusées dans le milieu où elles ont un effet néfaste sur la biodiversité. La forte utilisation des fourrages et du pâturage, et la diversification des ressources fourragères (avec une place prépondérante des légumineuses) offrent des opportunités pour faire consommer aux animaux des plantes à tanins dont les effets ont été bien documentés vis-à-vis de la maîtrise du parasitisme chez les ovins (Hoste et al 2015). Par ailleurs, le mode d'utilisation des autres médicaments est inscrit dans le cahier des charges (nombre de traitements limité par animal, déclassement possible des carcasses, traitement par individu), en particulier celui des antibiotiques dont les usages systématiques parfois observés en élevage conventionnel peuvent favoriser des phénomènes de résistance et questionner à long terme la maîtrise de certaines pathologies humaines (Sautereau et Benoit 2016). En revanche, la position personnelle de certains éleveurs en $A B$ vis-à-vis de directives officielles concernant la gestion sanitaire des troupeaux pourrait avoir une incidence sur la gestion collective des risques. L'épisode de
Fièvre Catarrhale Ovine de 2007 en est un exemple, avec le refus de certains éleveurs de vacciner leur troupeau, ceci n'étant pas en lien avec la réglementation de l'AB qui autorise les vaccinations. Une généralisation de ce type d'attitude pourrait amener à questionner l'impact attendu des directives mises en place en cas d'épizootie (Rat-Aspert et al 2012).

La prise en compte du bien-être animal dans les cahiers de charges poulet LR et élevage $A B$, et en particulier la possibilité donnée aux animaux d'exprimer leurs comportements naturels, se traduit par une obligation d'accès des animaux à des surfaces extérieures, dont le pâturage pour les herbivores. Cette notion de bienêtre animal est aussi traduite en termes de densité animale à l'extérieur ou dans les bâtiments. Ainsi en production LR, les poulets disposent d'environ deux fois plus de surface par animal en bâtiment ; en outre, la taille des lots est plafonnée à quelques milliers d'individus contre plusieurs dizaines de milliers dans les systèmes conventionnels (tableau 1). À l'inverse, cet accès à des surfaces extérieures peut augmenter le risque parasitaire et de prédation (Stahl et al 2002, Miao et al 2005, Cabaret et Nicourt 2009).

\section{5 / Services socio-culturels}

Les cahiers des charges et les principes de ces deux productions sous label contribuent à l'image de produits de terroir. En effet, les cahiers des charges LR imposent un minimum de 50 à $75 \%$ de céréales (et coproduits de céréales ; tableau 1) dans l'alimentation des poulets avec très souvent un approvisionnement local en matières premières. Cela contribue à renforcer l'image d'un produit « traditionnel » attaché à son territoire (poulet nourri " au grain »). Ce lien entre produit et territoire est d'ailleurs d'autant plus affirmé que ces productions Label Rouge sont fréquemment associées à une Indication Géographique Protégée (34 IGP aujourd'hui pour les volailles de chair). En outre, de par leurs qualités gustatives, les volailles Label Rouge sont majoritairement consommées entières (Taconet et Magdelaine 2009). Ainsi environ $60 \%$ des poulets entiers achetés par les ménages sont Label Rouge (SYNALAF 2015), et consommés lors de repas « traditionnels » ou « festifs » qui renvoient aux aspects culturels et traditionnels de la gastronomie française (cf. le « repas gastronomique des Français » inscrit au patrimoine mondial de l'UNESCO en 2010).

En production ovine $\mathrm{AB}$, le lien entre produit, territoire et terroir passe par la production de ressources alimentaires locales (tableau 1), ainsi que, selon les 
préconisations, l'utilisation de génotypes adaptés aux conditions du milieu. Les éleveurs privilégient les races ovines locales, souvent d'effectifs réduits (par exemple Bizet, Raïole, Thônes et Marthod, Solognote, Rava...), ce qui contribue à la préservation de la biodiversité domestique, et renforce le lien au « terroir ». Les capacités adaptatives des animaux sont en effet déterminantes, et les éleveurs recherchent en priorité des races caractérisées par leur rusticité, mais aussi par leur capacité de « finition» des produits à l'herbe, leur résistance au parasitisme, leur aptitude à la marche, leur résistance au froid ou à l'humidité et leur autonomie de mise bas (Boissy et al 2005, Grandin et Deesing 2014). En revanche, ces races peuvent présenter une faible conformation qui pénalise la mise en marché, sauf si les éleveurs pratiquent des croisements avec des races à viande et/ou la vente en circuit court, leurs caractéristiques raciales ainsi que leurs liens aux terroirs étant appréciées par le consommateur.

Les élevages LR élèvent très majoritairement des souches commerciales mais le nombre total de souches disponibles pour cette production est nettement plus élevé que pour la production standard (une quarantaine contre moins d'une dizaine pour la production de poulets à croissance rapide, SYSAAF ${ }^{1}$ communication personnelle) en raison d'une plus grande diversité des produits issus de ces élevages (poulets à cou nu, couleur du plumage ou de la peau...). Les systèmes en Label Rouge contribuent ainsi à la conservation de la biodiversité domestique. Toutefois, les sélectionneurs, tant pour les souches utilisées en LR qu'en production conventionnelle, disposent d'un nombre bien plus élevé de lignées utilisables pour réaliser des croisements et proposer des souches adaptées à différents marchés. Cette diversité « masquée » (données confidentielles) contribue également à la conservation de la biodiversité domestique (i.e. réservoir de gènes ; SYSAAF communication personnelle).

Le respect de l'animal, qui conduit en particulier à lui permettre d'exprimer ses comportements naturels, ainsi que la moindre intensification de son cycle de production vis-à-vis de l'Agriculture Conventionnelle $(\mathrm{AC})$ conduisent à réduire l'efficience de production et renforcer les interactions de l'animal avec le milieu naturel. Ceci peut engendrer des impacts négatifs, par exemple en termes d'utilisation globale d'intrants (alimentation), d'impact sur les milieux naturels, de maîtrise sanitaire. Le chapitre suivant vise à analyser les leviers mobilisables pour limiter ces conséquences potentiellement négatives, optimiser les performances et renforcer les services fournis.

\section{2 / Quels leviers pour renfor- cer les services et réduire les antagonismes ?}

\section{1 / Recherche de compromis entre efficience de production, respect du cahier des charges et image des produits}

En production de poulets, l'amélioration de l'efficacité alimentaire permet de réduire l'utilisation d'intrants et leurs effets négatifs indirects sur l'environnement et les ressources (émissions de GES, gestion des déjections, consommation d'énergie). Différentes approches nutritionnelles déjà existantes pour d'autres espèces ou productions pourraient ainsi avoir un intérêt en production de poulets LR, par exemple l'alimentation séquentielle, qui permet, en outre, d'utiliser de façon directe des céréales produites sur l'exploitation (Umar Faruk et al 2010), ou encore l'alimentation de précision, qui permet d'apporter des rations parfaitement adaptées aux besoins des animaux (Andretta et al 2016), même si pour cette dernière approche, des efforts de recherche sont encore nécessaires pour l'adapter aux spécificités des volailles (gestion à l'échelle du lot, mesure régulière du poids et de la consommation, modèles de prédiction des besoins). Enfin, l'utilisation d'acides aminés de synthèse permet également d'envisager la réduction du taux protéique des rations distribuées aux poulets de chair, sans dégradation des performances et avec des conséquences positives, sur l'excrétion azotée notamment (- $13 \%$ par point de protéines, Belloir et al 2017).

En production ovine, la productivité numérique est un élément déterminant de la performance économique mais aussi de la limitation des émissions de GES par kg de viande produite, grâce à l'effet de dilution qu'elle permet sur le méthane entérique des brebis. Cependant, cet intérêt devient faible voire nul pour des niveaux très élevés nécessitant des niveaux d'intrants (dont les concentrés) très importants, un parallèle pouvant être fait avec la production laitière (Gerber et al 2011). Une productivité numérique très élevée peut poser des problèmes de maîtrise sanitaire (Benoit et al 2009), de travail (volume de production, surveillance...). Des optimums définis selon les contextes existent donc, qui prennent la forme de compromis entre performance économique, satisfaction de la filière (régularité d'approvisionnement), charge de travail, niveau d'émission de méthane, et santé animale.

\section{2 / Réduire l'utilisation d'intrants}

a) La question centrale de l'alimentation

L'utilisation d'intrants, dont les concentrés, a diverses incidences négatives, en particulier d'un point de vue environnemental (pollutions diffuses et/ou perte de biodiversité et/ou incidences sur le changement climatique).

Les pratiques associées à l'alimentation sont un levier majeur d'amélioration des diverses performances, en élevage ovin, en renforçant les capacités de valorisation des ressources locales. L'objectif est de faire coïncider les besoins des animaux et les disponibilités en herbe de qualité. Cela peut conduire à concentrer les mises-bas au printemps, pour des ventes d'agneaux regroupées entre les mois de juin et de novembre. Le contexte pédoclimatique des fermes oriente ainsi souvent l'organisation du système de production, la réponse au marché venant dans un second temps (Benoit et Laignel 2009, Coquil et al 2009), ce qui peut imposer de fortes capacités d'adaptation aux opérateurs des fillières.

Un niveau très élevé d'autonomie fournie par les fourrages peut représenter un fort handicap vis-à-vis des aléas climatiques dont les conséquences peuvent être graves d'un point de vue économique. L'une des parades peut consister à constituer un volant de sécurité important en termes de stocks fourragers afin de tamponner la variabilité de la disponibilité des ressources fourragères. La diversification des activités, fréquemment pratiquée en $\mathrm{AB}$ (multi-espèces animales ou végétales), est également très favorable à la résilience vis-à-vis d'aléas climatiques et économiques.

La réduction de l'usage des concentrés peut être envisagée en augmentant la qualité de l'herbe pâturée, ce qui peut être réalisé par l'adoption de pratiques telle le pâturage cellulaire (Voisin 2001), la récolte précoce de fourrages pour favoriser les repousses estivales, le sur-semis de mélanges associant des légumineuses et d'autres dicotylédones, qui peut par ailleurs permettre de créer plus rapidement des prairies « multifonctionnelles » (Bradbury et al 2010). Néanmoins, afin de contrebalancer les conséquences de récoltes précoces (moindre infloraison, moindre biodiversité des insectes, voire moindre nidification pour certains oiseaux), l'utilisation de certaines par- 
celles en pâturage ou en fauche peut être retardée (Farruggia et al 2012).

En production avicole, la question de l'autonomie protéique et de la dépendance au soja importé est centrale. De par une vitesse de croissance réduite, les besoins en protéines des poulets LR sont plus faibles qu'en production conventionnelle : la réduction totale ou partielle du tourteau de soja dans les aliments est plus facilement envisageable, comme montré par Méda et al (2015), en utilisant des protéagineux (pois, féverole) et davantage de coproduits de céréales et de tourteaux d'oléagineux. Ce changement d'alimentation a peu de conséquences sur le coût de production et sur la qualité de la viande mais permet des gains environnementaux importants, avec par exemple une réduction de $40 \%$ des émissions de GES.

En production ovine, le pâturage de surfaces pastorales en hiver est favorable à la limitation des coûts d'alimentation et de l'énergie fossile utilisée et entre pleinement dans la notion de lien au sol. Cependant, ces pratiques peuvent se heurter à la présence de grands prédateurs engendrant de fortes contraintes pour les éleveurs, en particulier en termes de travail et de charge mentale, et générant un coût élevé pour la collectivité. D'autres prédateurs plus courants peuvent pénaliser les élevages dans lesquels les mises bas ont lieu à l'extérieur ou pour l'élevage de poulets LR (renards, corvidés, rapaces...), avec une régulation possible (chiens de protection, filets, agnelages en bergerie aux périodes sensibles).

\section{b) Limitation des intrants médicamenteux et maitrise du parasitisme}

L'AB privilégie une vision préventive de la santé animale. Le nombre global de traitements sanitaires par animal peut baisser significativement en comparaison à l'élevage conventionnel (deux fois moins, en production bovin lait et viande ; Pavie et al 2012), ce qui peut bénéficier aux performances économiques et réduit les résidus médicamenteux dans les produits et l'environnement. Cependant, l'engraissement au pâturage des agneaux fait surgir un enjeu majeur : les ovins, dont les jeunes, ont une forte sensibilité aux parasites, qui affectent les performances techniques (croissance des agneaux, mortalité) voire environnementales (GES/kg de carcasse par exemple) et peuvent conduire à de la mortalité.

Des leviers existent cependant, dans une démarche globale construite autour de trois notions : «Éviter », « Résister », « Traiter». Ces items se déclinent en divers leviers visant à réduire les interactions entre hôtes et pathogènes (via, en particulier, le réajustement du système de production), utiliser les plantes bioactives et d'autres méthodes alternatives, et définir des indicateurs et des seuils d'intervention en accord avec des niveaux de perte acceptables (Hoste et al 2009). La réduction du nombre de traitements pose la question du niveau de souffrance que la pathologie ou les niveaux parasitaires font subir à l'animal, sachant qu'un bon état de santé de l'animal fait partie des quatre principes fondamentaux de l'AB et qu'il est directement associé au bien-être de l'animal (Bellon et al 2009). La réduction de l'utilisation des produits chimiques de synthèse pour la maîtrise de la santé peut nécessiter la mise en place d'un système d'observation des animaux, d'indicateurs de suivi plus complexes pour des interventions moins systématiques et au cas par cas, conduisant à des contraintes de temps et d'organisation. Elle peut aussi reposer sur l'utilisation de races ou souches plus robustes ou sur l'ingestion par les animaux de plantes à effet « médicinal » (Hoste et al 2015, Zeng et al 2015), même si l'opérationnalité de cette hypothèse reste à valider chez les volailles (Germain et al 2015).

\section{3 / Diversifier pour renforcer la résilience des systèmes et les services fournis}

Le lien au sol conduit à une dépendance accrue des ressources disponibles vis-à-vis des aléas climatiques. Ce risque peut être contourné par la diversification qui permet d'une part de stabiliser les processus écologiques (Dumont et al 2013), d'autre part de limiter les risques économiques en misant sur des filières différenciées. L'association de poulets LR à des productions de fruits ou de bois dans des systèmes " agroforestiers 》 va dans ce sens (Lubac et al 2003, Dubois et al 2008).

Cette diversification des activités (multiespèces animales ou végétales) est par ailleurs bien adaptée au développement de filières courtes (notion d'économie de gamme) et peut être antagoniste aux filières longues, avec des coûts de collecte élevés du fait d'une fragmentation des livraisons avec de faibles volumes. Elle est tout à fait cohérente avec le rapprochement producteur-consommateur qui rentre dans les principes du mode de production $\mathrm{AB}$ par exemple. Cependant, elle requiert une maitrise technique des différentes productions et une technicité accrue pour prendre en compte les interactions techniques et organisationnelles entre ateliers. Par ailleurs, certains outils d'aval (abattoirs par exemple) sont absents de certains territoires très spécialisés (grandes cultures par exemple) ce qui limite les possibilités de vente en circuit court.

\section{4 / Conforter l'acceptabilité sociale}

a) Conduites «traditionnelles » et mutilations

Les mutilations sont des pratiques traditionnellement utilisées, en général soit pour compenser les effets provoqués par des modes d'élevage intensifs ou confinés (épointage du bec, édentage, caudectomie...), soit pour améliorer la qualité des produits ou faciliter la conduite d'élevage.

Les mutilations sont encadrées en $\mathrm{AB}$, mais autorisées sous certaines conditions. La dérogation octroyée par exemple pour la castration facilite l'engraissement à l'herbe, phase pendant laquelle intervient la maturité sexuelle des agneaux mâles qui conduit à des modifications des comportements : elle simplifie la conduite en permettant le mélange des agneaux mâles et femelles et limite les problèmes de qualité de carcasse redoutés par la filière (Normand et al 2000).

b) Renforcer l'image «terroir » via le lien au sol de l'alimentation

L'origine de l'alimentation des animaux est une question centrale en production de poulets LR. La suppression intégrale du tourteau de soja, qui permet de proposer une alimentation garantie sans OGM et responsable (non associée à de la déforestation par exemple), peut être envisagée avec la substitution par des matières premières locales (pois, féverole, tourteau de colza...), plus facile en production LR en raison des besoins protéiques plus faibles des animaux à croissance lente (Méda et al 2015). L'autonomie et le lien au territoire sont ainsi renforcés, renvoyant une image positive au consommateur.

L'élevage sur parcours jouit également d'une image positive auprès des consommateurs, que ce soit en production ovine ou de poulets LR. Il permet aux animaux d'exprimer leurs comportements spécifiques, un élément qui sous-tend la notion de bien-être. Cependant, l'accès au parcours en production de poulets LR pose aussi la question de la sécurité sanitaire pour les consommateurs. Outre les risques de mortalité liés à la prédation, les animaux sont susceptibles d'être en contact avec la faune sauvage (et les déjections) porteuse de maladies ou de pathogènes (van de Weerd et al 2009, Lubac et al 2012). Enfin, si les sols et la végétation des parcours contiennent des contaminants chimiques environnementaux (PCB, dioxines...), l'exposition des animaux y ayant accès est supérieure à celle d'animaux élevés en claustration (Jondreville et al 2014). Les plans de surveillance de la DGAL montrent tou- 
tefois que la contamination des produits (viande, œufs) au-delà des limites réglementaires demeure aujourd'hui très faible, et qu'elle est souvent associée à des contextes particuliers (accidents industriels par exemple).

\section{5 / Des leviers réfléchis et orga- nisés à une échelle collective}

De nombreux leviers peuvent être envisagés à une échelle collective, qu'ils touchent aux ressources utilisées, à la maitrise de la santé des cultures et des animaux, aux débouchés pour les produits, aux échanges de savoirs.

L'utilisation de génotypes animaux adaptés peut permettre de maximiser les services fournis, grâce à leur potentiel adaptatif. Cependant, malgré la diversité encore présente de génotypes "rustiques " adaptés, les effectifs de leurs populations sont parfois faibles et nécessitent une gestion collective visant en particulier à garder le maximum de variabilité génétique. Pour ce qui concerne les gestions des ressources alimentaires, de la fertilisation des cultures et de la bonne maîtrise globale des cycles des minéraux, elles pourraient être optimisées par des organisations à l'échelle des territoires à travers l'échange de matières premières entre éleveurs et céréaliers (transferts d'aliments et de paille, restitution des fumiers). Ryschawy et al (2017, ce numéro) développent les leviers mobilisables à l'échelle des territoires dans cette optique. La gestion de la santé des cultures associées à ces élevages pourra profiter d'une organisation collective au travers de la mise en place d'infrastructures paysagères favorisant les corridors écologiques et la maximisation des mécanismes de régulation biologique (Sabatier et al 2014). La maîtrise des épizooties peut également nécessiter des concertations permettant de satisfaire à la fois aux objectifs de maitrise globale et aux cahiers des charges et principes des productions sous SIQO. Pour ce qui est de la gestion de la mise en marché des produits, compte tenu des objectifs de connexion entre besoins des animaux, disponibilité des ressources saisonnières et des contextes pédoclimatiques variables des fermes, elle devra faire l'objet de concertation et de planification.

Enfin, il est reconnu que la maîtrise des systèmes de production en $\mathrm{AB}$ nécessite des connaissances techniques importantes mais surtout d'aborder les systèmes de production dans leur globalité et leur complexité. Par ailleurs, les références en $\mathrm{AB}$ sont beaucoup moins nombreuses que celles disponibles en AC. Globalement, les producteurs $\mathrm{AB}$ sont plus formés que leurs homologues en AC. Ils utilisent les voies classiques d'information mais aussi des dispositifs spéci- fiques, comme les groupements d'agriculteurs en $A B(G A B)$ qui privilégient le transfert de savoir au travers de groupes d'échanges (Sautereau 2009). Pour la production de poulets LR, la maitrise des itinéraires techniques passe avant tout par l'appui technique qui est apporté aux éleveurs par les organisations de production.

\section{3 / Analyse générique de la production de services en production sous labels}

Les notions d'autonomie alimentaire et de lien au sol sont centrales pour les productions étudiées, et peuvent contribuer à améliorer leurs performances économiques et environnementales. La production de tout ou partie de l'alimentation sur la ferme permet de boucler les cycles des minéraux (retour des déjections) tout en limitant les transports de matières premières. En élevage d'herbivores, elle passe avant tout par la maximisation de l'utilisation des fourrages, en particulier via le pâturage. La recherche d'une adéquation forte entre les besoins des animaux et les ressources saisonnières disponibles, en particulier pour les herbivores au pâturage, conditionne le calendrier de mise en marché des produits, ce qui nécessite une coordination avec les acteurs de la filière et une planification collective.

L'autonomie alimentaire questionne en particulier l'origine des protéines, avec la nécessité de disposer de protéagineux pour assurer la croissance des animaux. Leur culture locale, et si possible à la ferme, est un atout, voire une condition. Aussi, ces deux productions (ovins en AB et poulets LR) ont-elles une position singulière vis-à-vis de la construction de systèmes polycultureélevage en permettant d'optimiser les cycles des minéraux (échanges fumiers/ paille/aliments). La présence d'herbivores peut encourager la mise en place et la valorisation de rotations longues, en particulier en $\mathrm{AB}$, incluant des cultures fourragères, en dérobée ou non, bien valorisées par les animaux, en particulier aux saisons intermédiaires, voire en hiver. Indirectement, ces rotations contribuent à la réduction des intrants (engrais et pesticides) (Ryschawy et al 2017).

Dans ces modes de production sous signe officiel de qualité, la limitation des niveaux de croissance des animaux procure aux produits des spécificités organoleptiques et nutritionnelles favorables (sauf exception pour le scatole éventuel en production ovine) et une image favorable pour le consommateur (produit « traditionnel», gastronomie). Cependant, la moindre efficacité alimen- taire peut peser sur les indicateurs environnementaux, exprimés par unité de produit (émissions de GES, consommation d'énergie) du fait de la moindre dilution de certaines composantes du bilan (méthane par exemple). Néanmoins, des compensations (moins de $\mathrm{N}_{2} \mathrm{O}$ en l'absence d'engrais azotés de synthèse par exemple), permettent de maintenir le niveau des indicateurs environnementaux au niveau des références, voire de leur donner un avantage, surtout si l'on utilise des unités fonctionnelles spécifiques (unité de surface ou $€$ de produit). Par ailleurs, le moindre chargement animal par ha (ovins) et la part importante de surfaces de prairies peuvent accroitre la contribution du carbone stocké dans le sol, rapporté au $\mathrm{kg}$ de viande. En revanche, la surface agricole nécessaire par $\mathrm{kg}$ de viande produite est supérieure aux standards.

Les cahiers des charges favorisent le bien-être animal (accès extérieurs, moindres mutilations, surface disponible par animal...) et contribuent ainsi à la différenciation de ces produits vis-àvis des standards, assurant un niveau de valorisation supérieur, en adéquation avec un consentement à payer supérieur des consommateurs (e.g. pour des poulets ayant accès à un parcours; Mulder et Zomer 2017). En particulier, l'utilisation des parcours a des effets positifs vis-à-vis du bien-être animal et de la biodiversité, voire sur les émissions de GES (via la séquestration du carbone) et dans la représentation du consommateur, mais aussi des effets potentiellement négatifs sur la santé animale (parasitisme) et sur la productivité (mortalité d'animaux via la prédation).

La diversification des systèmes de production visant à stimuler les processus d'autorégulation pour maximiser l'autonomie génère une complexité de pilotage et un surcroit de travail, qui, dans le cadre de l'AB, est exacerbée par l'adaptation des conduites aux contextes. La diversité des cultures dans des rotations longues (céréales, protéagineux) peut non seulement contribuer à la fourniture d'une ration équilibrée mais aussi améliorer la résilience des cultures vis-à-vis des maladies et ravageurs (en limitant le recours aux pesticides) et être source de biodiversité. La diversité des systèmes observée est basée sur les combinaisons variées entre élevage et cultures, mais aussi sur la combinaison de divers ateliers d'élevages. Associer plusieurs espèces animales pour bénéficier des synergies permises par la mixité (Dumont et al 2013) permet en effet une meilleure utilisation des couverts pâturés et donc de meilleures performances animales (d'Alexis et al 2014), une diminution de la pression parasitaire qui s'exerce sur les animaux par effet de dilution (non pathogénicité 
des larves ingérées par une espèce non compatible) et une diversification des productions qui tamponne les résultats économiques globaux. La diversité des systèmes peut aussi reposer sur celle des races utilisées, avec la recherche d'une bonne adaptation aux conditions du milieu et de leur efficacité de valorisation de ses ressources. Cette diversité est aussi un élément favorable vis-à-vis du consommateur (lien au terroir...) et participe à la conservation d'une certaine biodiversité « domestique ». Cependant, d'une part les productivités de ces génotypes animaux peuvent être inférieures à celles de génotypes classiquement utilisés, et d'autre part, les produits commercialisés ne répondent pas forcément aux normes habituelles de mise en marché, comme la conformation des carcasses par exemple en ovins $A B$, ce qui nécessite une concertation avec les opérateurs des filières.

\section{Conclusion}

Les productions sous signes officiels de qualité étudiées ici font l'objet d'une forte reconnaissance par les consommateurs, en relation avec les éléments constituant leurs cahiers des charges, les pratiques mises en œuvre et les caractéristiques de leurs produits. Les services fournis sont contrastés mais globalement très positifs. Le handicap majeur provient des moindres productivités animales et efficacités alimentaires qui in fine accroissent les besoins en ressources (dont la surface agricole), pour un volume de production donné. Cependant, cette moindre productivité et les génotypes utilisés procurent des propriétés favorables aux produits, du point de vue organoleptique mais aussi éthique (bienêtre animal) et patrimonial. Ces systèmes de production sont largement fondés sur l'utilisation de ressources locales ce qui optimise les bilans environnementaux. Les modes de production (ateliers de taille limitée) et les plus-values procurées par la bonne valorisation de ces produits se traduisent par des impacts socio-économiques territoriaux favorables. La mise en œuvre des principes de l'agroécologie dans les systèmes en $\mathrm{AB}$ permet d'exacerber les impacts environnementaux favorables (limitation des intrants polluants, maintien de biodiversité), en particulier via la diversification des activités intra ferme. Néanmoins, cette démarche conduit à la complexification des systèmes de production, à des solutions contextualisées, et à plus de technicité.

Parmi les enjeux majeurs pour le développement de ces productions, nous pouvons retenir la nécessité d'améliorer l'efficacité alimentaire et la productivité de l'animal (dont les questions liées à la santé-parasitisme et à la prédation), et la maximisation de l'utilisation des services écosystémiques (fixation d'azote, gestion des ravageurs par les régulations biologiques).

Par ailleurs, ces modes de production dessinent de nouveaux optimums entre niveaux de productivité et production de services (dont les caractéristiques des produits mis en marché) et mettent un focus sur la réflexion centrale à avoir autour des ressources locales utilisées (en particulier pour les ruminants) ainsi que sur les enjeux collectifs de l'optimisation des systèmes à l'échelle des territoires. Des dynamiques à ce niveau sont largement dépendantes des organisations existantes ou à créer et des soutiens publics. Enfin, ces modes de production, fondés sur de nouvelles relations entre producteurs et consommateurs/citoyens, peuvent être source de réflexion pour orienter les évolutions des modes de production plus intensifs, bon nombre de pratiques mise en évidence précédemment étant d'ailleurs souvent reprises dans des systèmes de production conventionnels à la recherche de nouveaux optimums passant par la réduction des charges de production.

\section{Références}

Agence Bio, 2016a. La bio dans l'Union Européenne. Édition 2016. 40p.

Agence Bio, 2016b. La bio dans les territoires. Fiches régionales et de production. Édition 2016. $76 \mathrm{p}$.

Andretta I., Pomar C., Rivest J., Pomar J., Radünz J., 2016. Precision feeding can significantly reduce lysine intake and nitrogen excretion without compromising the performance of growing pigs. Animal, 10, 1137-1147.

Aurousseau B., Bauchart D., Calichon E., Micol D., Priolo A., 2004. Effect of grass or concentrate feeding systems and rate of growth on triglyceride and phospholipid and their fatty acids in the M-longissimus thoracis of lambs. Meat Sci., 66, 531-541.

Bellet V., Morin E., 2005. Approche des coûts de production et des déterminants du revenu en élevage ovin viande. Compte-rendu final 1105 50 020. Éditions Institut de l'Élevage, 73p.

Bellet V., Experton C., Gac A., Laignel G., Morin E. 2016. Les systèmes ovins biologiques sont-ils plus durables que les conventionnels? Renc. Rech. Rum., 23, 243-246.

Belloir P., Méda B., Lambert W., Corrent E., Juin H., Lessire M., Tesseraud S., 2017. Reducing the crude protein content in broiler feeds: Impact on animal performance, meat quality and nitrogen utilization. Animal, 11, 1881-1889.
Bellon S., Prache S., Benoit M., Cabaret J., 2009. Recherches en élevage biologique enjeux, acquis et développements. In : Numéro spécial, Élevage bio. Perez J.M. (Éd). INRA Prod. Anim., 22, 271-284.

Benoit M., Laignel G., 2009. Performances techniques et économiques en élevage biologique d'ovins viande : observations en réseaux d'élevage et fermes expérimentales. In : Numéro spécial, Élevage bio. Perez J.M. (Éd). INRA Prod. Anim., 22, 197-206.

Benoit M., Dakpo H., 2012. Greenhouse gas emissions on french meat sheep farms: analysis over the period 1987-2010. In: Intern. Symp. Émis. Gas and Dust from Livest.. Hassouna M., Guigand N. (Eds). EmiLi 2012, Saint-Malo, France, 384-387.

Benoit M., Laignel G., Lienard G., 1999 Facteurs techniques, cohérence de fonctionnement et rentabilité en elevage ovin allaitant. Exemples du Massif central Nord et du Montmorillonnais. Renc. Rech. Rum., 6, 19-22.

Benoit M., Tournadre H., Dulph J.P., Laignel G., Prache S., Cabaret J., 2009. Is intensification of reproduction rhythm sustainable in an organic sheep production system? A 4-year interdisciplinary study. Animal, 3, 753-763.

Benoit M., Laignel G., Roulenc M., 2010. Émissions de gaz à effet de serre et consommations d'énergie en élevage ovin viande. Renc. Rech. Rum., 17, 351-354.
Boissy A., Bouix J., Orgeur P., Poindron P., Bibé B., Le Neindre P., 2005. Genetic analysis of emotional reactivity in sheep: effects of the genotypes of the lambs and of their dams. Genet. Sel. Evol., 37, 381-401.

Bourguet D., Guillemaud T., 2016. The hidden and external costs of pesticide use. In: Sustain. Agricult. Revi., 19. Lichtfouse E. (Ed). Springer, Berlin, Allemagne, 35-120.

Bradbury R.B., Stoate C., Tallowin J.R.B., 2010. FORUM: Lowland farmland bird conservation in the context of wider ecosystem service delivery. J. Appl. Ecol., 47, 986-993.

Cabaret J., Nicourt C., 2009. Les problèmes sanitaires en élevage biologique : réalités, conceptions et pratiques. In : Numéro spécial, Élevage bio. Perez J.M. (Éd). INRA Prod. Anim., 22, 235-243.

Chartrin P., Berri C., Lebihan-Duval E., Quentin M., Baeza E., 2005. Lipid and fatty acid composition of fresh and cured-cooked breast meat of standard, certified and label chickens. Arch Geflugelkd, 69, 219-225.

Chevalier D., Nicolas C., Amand G., 2015. Le bâtiment d'élevage, un outil stratégique pour la filière avicole. Journ. Rech. Avicole et Palmipèdes à Foie Gras, 11, 840-854.

Clark M.S., Gage S.H., 1996. Effects of freerange chickens and geese on insect pests and weeds in an agroecosystem. Am. J. Alternative Agr., 11, 39-47. 
Coquil X., Blouet A., Fiorelli J.L., Bazard C., Trommenschlager J.M., 2009. Conception de systèmes laitiers en agriculture biologique : une entrée agronomique. In : Numéro spécial, Elevage bio. Perez J.M. (Ed). INRA Prod. Anim., 22, 221-234.

D'Alexis S., Sauvant D., Boval M., 2014 Mixed grazing systems of sheep and cattle to improve liveweight gain: a quantitative review. J. Agricult. Sci., 152, 655-666.

da Silva V.P., van der Werf H.M.G., Soares S.R., Corson M.S., 2014. Environmental impacts of French and Brazilian broiler chicken production scenarios: An LCA approach. J. Environ. Manage., 133, 222-231.

Dekker S.E.M., Aarnink A.J.A., De Boer I.J.M., Groot Koerkamp P.W.G., 2012. Total loss and distribution of nitrogen and phosphorus in the outdoor run of organic laying hens. Br. Poultr. Sci., 53, 731-740.

Dubois J.P., Bijja M., Auvergne A., Lavigne F., Fernandez X., Babilé R., 2008. Agroforesterie : comportement des oies sous un couvert de noyers et effets sur les performances du verger. Journ. Rech. Palmipèdes à Foie Gras, 8, 111-115.

Dumont B., Fortun-Lamothe L., Jouven M., Thomas M., Tichit M., 2013. Prospects from agroecology and industrial ecology for animal production in the $21^{\text {st }}$ century. Animal, 7,1028 1043.

Duru M., Donnars C., Ryschawy J., Therond O., Dumont B., 2017. La « grange » un cadre conceptuel pour appréhender les bouquets de services rendus par dans les territoires. In : Numéro spécial, L'élevage en Europe : une diversité de services et d'impacts. Dumont B (Éd). INRA Prod. Anim. 30, 273-284.

Fanatico A.C., Pillai P.B., Emmert J.L., Owens C.M., 2007. Meat quality of slow- and fastgrowing chicken genotypes fed low nutrient or standard diets and raised indoors or with outdoor access. Poult. Sci., 86, 2245-2255.

Farruggia A., Dumont B., Scohier A., Leroy T., Pradel P., Garel J.P., 2012. An alternative rotational stocking management designed to favour butterflies in permanent grasslands. Grass Forage Sci., 67, 136-149.

Garambois N., Devienne S., 2010. Évaluation économique, du point de vue de la collectivité, des systèmes bovins laitiers herbagers. Renc. Rech. Rum., 17, 25-32.

Gerber P.J., Vellinga T., Opio C., Steinfeld H., 2011. Productivity gains and greenhouse gas emissions intensity in dairy systems. Livest. Sci., 139, 100-108.

Germain K., Guesdon V., Cayez C., Lamothe E., Cabaret J., 2015. L'implantation de plantes médicinales sur les parcours de volailles biologiques : quels intérêts, quelles conséquences? Journ. Rech. Avicole et Palmipèdes à Foie Gras, $11,220-224$

Grandin T., Deesing M.J., 2014. Chapter 1 Behavioral Genetics and Animal Science. In: Genetics and the Behavior of Domestic Animals (Second Edition). Grandin T., Deesing M.J. (Eds). San Diego: Academic Press, San Diego, USA, 1-40.

Hoste H., Cabaret J., Grosmond G., Guitard J.P., 2009. Alternatives aux traitements anthelminthiques en élevage biologique des ruminants. In : Numéro spécial, Élevage bio. Perez J.M. (Éd). INRA Prod. Anim., 22, 245-254.
Hoste H., Torres-Acosta J.F.J., SandovalCastro C.A., Mueller-Harvey I., Sotirakie S., Louvandini H., Thamsborg S.M., Terrill T.H., 2015. Tannin containing legumes as a model for nutraceuticals against digestive parasites in livestock. Vet. Parasitol., 212, 5-17.

IFOAM, 2015. Principles of organic agriculture, 4p. http://www.ifoam.bio/sites/default/files/ poa_english_web.pdf

Jondreville C., Lavigne A., Jurjanz S., Dalibard C., Liabeuf J.M., Clostre F., Lesueur-Jannoyer M., 2014. Contamination of free-range ducks by chlordecone in Martinique (French West Indies): A field stufy. Sci. Total Environ., 493, 336-341.

Kratz S., Rogasik J., Schnug E., 2004. Changes in soil nitrogen and phosphorus under different broiler production systems. J. Environ. Qual., 33, 1662-1674.

Laignel G., Benoit M., 2004. Production de viande ovine en agriculture biologique comparée à l'élevage conventionnel : résultats technicoéconomiques d'exploitations de plaine et de montagne du nord du Massif central. INRA Prod. Anim., 17, 133-143.

Lavigne A., Dumbardon-Martial E., Lavigne C., 2012. Les volailles pour un contrôle biologique des adventices dans les vergers. Fruits, 67, 341-351.

Lubac S., Senecaille M., Spérandio D., Desquennes A., Arnould C., Faure J.M., Mirabito L., 2003. Influence de la présence de taillis de saule à très courte rotation sur la fréquentation des parcours par les poulets Label. Journ. Rech. Avicole, 5, 77-80.

Lubac S., Musseau R., Caparros O., Artois M., Bicout D.J., 2012. Interactions entre l'avifaune sauvage et les élevages de volailles : quel risque épidémiologique vis à vis de l'Influenza aviaire ? Innov. Agro., 25, 299-312.

Lubac S., Roinsard A., Dartois S., Pourteau M., Beral C., Germain K., Bourgade E., Guillet P., 2014. Aménagements des parcours de poulets Label Rouge et Biologiques. TeMA: techniques et marchés avicoles, 30, 24-31.

Massis D., Hild F., 2016. La pratique de l'agriculture biologique créatrice d'emploi ? Évaluation de l'impact du bio sur la quantité de travail agricole. Agreste Les Dossiers, 35, 30p.

Méda B., Flechard C.R., Germain K., Robin P., Walter C., Hassouna M., 2012. Greenhouse gas emissions from the grassy outdoor run of organic broilers. Biogeosciences, 9, 1493-1508.

Méda B., Dusart L., Lessire M., Hallouis J.M., Bordeau T., Souchet C., Mercerand F., Hatte C., Bouvarel I., Berri C., 2015. Remplacement du tourteau de soja par des matières premières riches en protéines : Évaluation multicritère chez le poulet de chair de type Label Rouge. Journ. Rech. Avicole et Palmipèdes à Foie Gras, 11, 1082-1087.

Miao Z.H., Glatz P.C., Ru Y.J., 2005. Free-range poultry production - A review. Asian-Australa. J. Anim., 18, 113-132.

Mulder M, Zomer S., 2017. Dutch consumer's willingness to pay for broiler welfare. J. Appl. Anim. Welfare Sci., 20, 137-154.

Normand J., Pottier E., Sagot L., Brouard S., 2000. Quelques éléments techniques pour produire de l'agneau de report. Renc. Rech. Rum., 7, 268.
Pavie J., Chambaut H., Mousset E., Leroyer J., Simonin V., 2012. Évaluations et comparaisons des performances environnementales, économiques et sociales des systèmes bovins biologiques et conventionnels dans le cadre du projet CedABio. Renc. Rech. Rum., 19, 37-40.

Pottier E., Tournadre H., Benoit M., Prache S. 2009. Maximizing the share of grazing in the feeding of sheep : advantages for feed self-sufficiency, for the environment, and for the quality of the products. Fourrages, 199, 349-371.

Prache S., Ballet J., Jailler R., Meteau B., Picard B., Renerre D., Bauchart D., 2009. Comparaison des qualités de la viande et de la carcasse d'agneaux produits en élevage biologique ou conventionnel. Innov. Agro., 4, 289-296.

Protino J., Magdelaine P., Berri C., Méda B., Ponchant P., Dusart L., Chevalier D., Dezat E., Lescoat P., Bouvarel I., 2015. OVALI : Une méthode d'évaluation de la durabilité des filières avicoles 1-Utilisation pour évaluer l'existant et identifier des marges de progrès. Journ. Rech. Avicole et Palmipèdes à Foie Gras, 11, 10471053.

Rat-Aspert O., Petit E., Fourichon, C., 2012. La maîtrise collective de la santé animale à l'épreuve des choix individuels : approche théorique de la décision de vaccination des animaux. Renc. Rech. Rum.. 47-50.

Reganold J.P., Wachter J.M., 2016. Organic agriculture in the twenty-first century. Nat. Plants, 2, 15221 .

Ryschawy J., Benoit M., Hostiou N, Delfosse C., 2017. Quelles concurrences et synergies entre cultures et élevage dans les territoires de polyculture-élevage? In : Numéro spécial, L'élevage en Europe : une diversité de services et d'impacts. Dumont B. (Éd). INRA Prod. Anim. 30, 363-380.

Sabatier R., Doyen L., Tichit M., 2014. Heterogeneity and the trade-off between ecological and productive functions of agro-landscapes: A model of cattle-bird interactions in a grassland agroecosystem. Agricult. Sys., 126, 38-49.

Sautereau N. 2009. Soutenir le développement de l'AB : conseils et dispositifs incitatifs à la conversion. In transitions vers d'agriculture biologique, Éditions QUAE-Educagri, coord Lamine et Bellon., 193-218.

Sautereau N., Benoit M., 2016. Quantifier et chiffrer économiquement les externalités de l'agriculture biologique ? Rapport à la demande du Ministère de l'Agriculture, de l'Agroalimentaire et de la Forêt (MAAF). Rapport d'étude ITAB. 136p.

Sauveur B., 1997. Les critères et facteurs de la qualité des poulets Label Rouge INRA Prod. Anim., 10, 219-226.

Siegford J.M., Powers W., Grimes-Casey H.G. 2008. Environmental aspects of ethical animal production. Poult. Sci., 87, 380-386.

Srednicka-Tober D., Baranski M., Seal C., Sanderson R., Benbrook C., Steinshamn H., Gromadzka-Ostrowska J., Rembiałkowska E., Skwarło-Sońta K., Eyre M., Cozzi G., Krogh Larsen M., Jordon T., Niggli U., Sakowski T., Calder P.C., Burdge G.C., Sotiraki S., Stefanakis A., Yolcu H., Stergiadis S., Chatzidimitriou E., Butler G., Stewart G., Leifert C., 2016. Composition differences between organic and conventional meat: a systematic literature review and meta-analysis. Br. J. Nutr., 115, 994-1011. 
Stahl P., Ruette S., Gros L., 2002. Predation on free-ranging poultry by mammalian and avian predators: field loss estimates in a French rural area. Mammal Rev., 32, 227-234.

SYNALAF 2015. L'aviculture Label Rouge et biologique - Bilan économique Année 2014, 25p.

Taconet A.E., Magdelaine P., 2009. Segmentation du marché de la volailles par les modes de valorisation : État des lieux et perspectives. Journ. Rech. Avicole, 8, 178-182.

Tavoularis G., Recours F., Hébel P., 2007. Perception de la qualité et des signes officiels de qualité dans le secteur alimentaire. Cahier de Recherche CREDOC, 236, 114p.
Umar Faruk M., Bouvarel I., Même N., Rideau N., Roffidal L., Tukur H.M., Bastianelli D. Nys Y., Lescoat P., 2010. Sequential feeding using whole wheat and a separate proteinmineral concentrate improved feed efficiency in laying hens. Poult. Sci., 89, 785-796.

Union Européenne, 2007. RÈGLEMENT (CE) No 834/2007 DU CONSEIL du 28 juin 2007 relatif à la production biologique et à l'étiquetage des produits biologiques. $38 \mathrm{p}$.

van de Weerd H.A., Keatinge R., Roderick S. 2009. A review of key health-related welfare issues in organic poultry production. World Poult. Sci. J., 65, 649-684.

Verbeke W., Viaene J., 2000. Ethical challenges for livestock production: Meeting consumer concerns about meat safety and animal welfare. J. Agr. Environ. Ethic., 12, 141-151

Voisin A., 2001. Productivité de l'herbe. Édition France Agricole, Paris, France, 432p.

Zeltner E., Hirt H., 2003. Effect of artificial structuring on the use of laying hen runs in a free-range system. Br. Poult. Sci., 44, 533-537.

Zeltner E., Hirt H., 2008. Factors involved in the improvement of the use of hen runs. Appl. Anim. Behav. Sci., 114, 395-408.

Zeng Z.K., Zhang S., Wang H.L., Piao X.S., 2015. Essential oil and aromatic plants as feed additives in non-ruminant nutrition: a review. $\mathrm{J}$ Anim. Sci. Biotechnol., 6, 7.

\title{
Résumé
}

La demande des consommateurs évolue vers des produits pour lesquels des garanties (qualité, bien-être animal, respect de l'environnement) sont données via des cahiers des charges encadrant les pratiques. Cet article étudie les services rendus par deux productions sous signe officiel de qualité : les poulets Label Rouge et l'élevage ovin allaitant en agriculture biologique. Leurs cahiers des charges présentent des éléments de convergence (restriction d'utilisation de certains intrants, accès à des surfaces extérieures...) qui peuvent conduire à une productivité plus faible et pénaliser certains impacts environnementaux exprimés par kg de produit (GES, pour les poulets par exemple). Les bilans finaux peuvent cependant se révéler meilleurs qu'en conventionnel, par divers effets de compensation et s'ils sont exprimés par $€$ de produit. Ces productions renvoient une image positive auprès du consommateur en termes de bien-être animal (faible densité animale, accès à un parcours), de qualité organoleptique des produits et de lien au " terroir " et au territoire. Se dessinent alors de nouveaux optimums entre niveaux de productivité et production de services. Des leviers sont proposés pour maximiser les services environnementaux, sociaux et culturels tout en augmentant le service « d'approvisionnement »; à l'échelle de l'animal, les principaux enjeux concernent l'amélioration de l'efficacité alimentaire, l'adaptation aux milieux et la productivité numérique (ovins). La mobilisation des principes de l'agroécologie doit permettre, à l'échelle de l'exploitation, de tirer parti du couplage entre espèces ou ateliers de production (animal, végétal), et, à l'échelle du territoire, d'imaginer de nouvelles complémentarités entre exploitations.

\begin{abstract}
Challenges and assets of official signs of quality in livestock production to meet societal expectations: the example of organic lamb and Label Rouge chicken productions

Consumer demand is increasing for products with strong warranties regarding animal welfare, environmental respect or organoleptic quality. These warranties are generally given through official specifications regarding farming practices. We describe here the services provided by two productions with official signs of quality: Label Rouge chickens and organic lambs. Similar aspects can be found in both productions: limitation of the use of some inputs, access to outdoor areas. These elements lead to a lower productivity that can penalise some environmental impacts if expressed per $\mathrm{kg}$ of product (e.g. for chickens). Final balances can, however, be better than conventional ones thanks to various offsetting effects and if expressed by $€$ of product. These productions return a positive image to the consumer regarding animal welfare, organoleptic quality or the link to the "terroir". New optima appear between productivity level and the provision of services. Some proposals are given to maximize environmental and socio-economic services while improving "provision" service. At the animal scale, the main issues are the improvement of feed efficiency, adaptation to local conditions, ewe productivity. The use of agroecology principles should help, at the farm scale, to couple vegetal and animal productions and, at the region scale, to imagine new synergies between farms.
\end{abstract}

BENOIT M., MÉDA B., 2017. Enjeux et atouts des productions animales sous signe officiel de qualité pour répondre aux attentes sociétales. In : Numéro spécial, L'élevage en Europe : une diversité de services et d'impacts. Dumont B. (Éd). INRA Prod. Anim., 381-394. 